\title{
Optimal Placement of Mesh Points in Wireless Mesh Networks*
}

\author{
Suk Yu Hui ${ }^{1}$, Kai Hau Yeung ${ }^{1}$, and Kin Yeung Wong ${ }^{2}$ \\ ${ }^{1}$ Department of Electronic Engineering, City University of Hong Kong \\ syhui@ee.cityu.edu.hk, eeayeung@cityu.edu.hk \\ ${ }^{2}$ Computer Studies Program, Macao Polytechnic Institute \\ kywong@ipm.edu.mo
}

\begin{abstract}
A strategic placement of mesh points (MPs) in a Wireless Mesh Network (WMN) is essential to maximize the throughput of the network. In this paper, we address the problem of MPs placement for throughput optimization in WMN with consideration of routing protocols. Specifically, we formulate the routing paths among the MPs and mesh routers (MRs), calculate the aggregated traffic at the MPs, and suggest the optimal positions for the MPs. Our study also compares the optimal performance of routing protocols by finding their corresponding optimal placements of MPs first.
\end{abstract}

Keywords: wireless mesh network, gateway placement, throughput optimization.

\section{Introduction}

In a Wireless Mesh Network (WMN) [1], mesh routers (MRs) connect among themselves to form a wireless backbone. Unlike the traditional wireless LANs that require all wireless access points connecting to the wired network, WMNs only require some MRs connecting to the wired network. Those MRs are serving as gateways to the Internet, and called mesh points (MPs).

Providing quality Internet access for clients is an important issue in WMN [2]. Packets originated by the clients to the Internet are routed among the MRs to reach one of the MPs. With the dynamic self-organization and self-configuration, MRs in the network automatically establish and maintain route among themselves [3]. They may relay traffic on behalf of the other MRs which are not within direct wireless transmission ranges of the MPs. This paradigm is similar to the routing process in wired network, but the radio capacity of a WMN is usually lower than that of the wired network. In order to support high bandwidth broadband services, it is desirable to utilize the limited capacity efficiently and optimize the throughput capacity of the WMN [4, 5].

The placement of MPs strongly affects the performance, such as throughput of a WMN. Since the MPs aggregates the traffic flows, if they are misplaced, they may become the bottlenecks of data transmission. Zhang and Tsang proposed an algorithm

* This work was supported by City University Strategic Research Grant numbered 7001941. 
to form an optimal topology to balance the traffic in the network [6]. However, this study can be used for a newly designed WMN, but not for a WMN which topology is given. On the other hand, the authors in [7] consider the placement of MPs in a given network topology. They focused on the impact of link capacity constraints, wireless interference, fault tolerance, and variable traffic demands, and designed a series of placement algorithms.

Li. et al. proposed a novel grid-based gateway deployment method to solve the problem of gateway placement for throughput optimization in WMN [8]. Specifically, they studied how to place a given number of MPs for WMN so that the total throughput achieved can be maximized. Their results show that the method achieves better throughput than both random deployment and fixed deployment method. As can be seen, a detailed planning in the placement of MPs is essential in increasing the system capacity effectively. However, this study does not take the existing routing protocols into account.

In WMNs, routing protocols are needed for MRs so that they can forward traffic to the appropriate MP (Internet gateway). Routing protocols for WMN have a common goal which is to achieve a higher network throughput. However, most of the existing studies on the performance of these protocols are evaluated under the condition of predefined topology, i.e., the placement of the MPs is fixed [9-12]. This cannot reflect the optimal throughput that the routing protocol can achieve. In this paper, we study optimal placement of MPs in WMN with respect to different routing protocols. Given a set of routing paths that are generated from a given routing protocol, our goal is to find the positions of the MPs that optimize the throughput capacity of the network.

The rest of the paper is organized as follows. In Section II, we present the model of WMNs and mathematically formulate the throughput optimization problem for a given network topology. We then give numerical examples in Section III. Section IV concludes the paper.

\section{Problem Formulation}

Consider that the traffic in WMNs is mainly transmitted to or from the Internet. Since the Internet access is commonly asymmetric (i.e., downlink traffic is more than the uplink one), the goal of our study is to find the optimal placement of MPs that maximizes the downlink throughput. In the following, we formulate the placement problem under two scenarios: 1). WMN with a single MP, and 2). WMN with multiple MPs.

\subsection{WMN with a Single MP}

Supposing that a WMN contains $N$ MRs (see Figure 1). We define a set $A$ in which each element denotes one of the MRs, or $A=\left\{a_{1}, a_{2}, \ldots, a_{n}, \ldots, a_{N}\right\}$.

These MRs are connected with one another by many wireless links to form a wireless backbone network. Each MR associates with some mobile clients. The MP denoted as $a_{e}$ is the MP which connects to the wired network and act as the gateway 


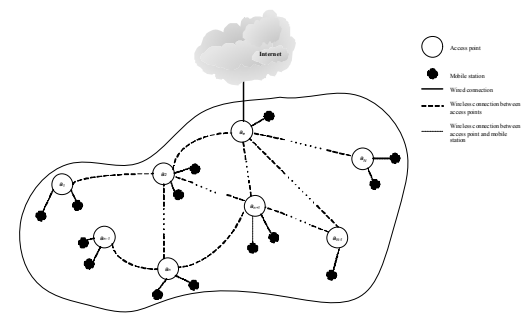

Fig. 1. A WMN with $\mathrm{N}$ mesh routers and one mesh point

for the network. In order to forward the data from the wired network to the mobile clients, multi-hopping routing is performed through the wireless backbone. There are a number of routing protocols for WMNs such as Routing Information Protocol (RIP), Hybrid Wireless Mesh Protocol (HWMP) and Radio Aware Optimized Link State Routing (RA-OLSR). Our method of finding the optimal placement of the MPs discussed below does not restrict the use of a particular routing algorithm. However, no multiple paths are allowed in our study.

Let matrix $C_{e}$ denotes the result of running the routing algorithm. It specifies the resulting paths from $a_{e}$ to each node.

$$
C_{e}=\left\{\begin{array}{ccccc}
c_{1,1} & c_{1,2} & \cdots & \ldots & c_{1, N} \\
c_{2,1} & \ddots & & & \vdots \\
\vdots & & c_{i, j} & & \vdots \\
\vdots & & & \ddots & c_{N 11, N} \\
c_{N, 1} & \cdots & \cdots & c_{N, N 11} & c_{N, N}
\end{array}\right)
$$

where

$$
c_{i, j}= \begin{cases}1 & \text { if } a_{i} \text { to } a_{j} \text { is a path from } a_{e} \text { to } a_{j} \\ 0 & \text { if } a_{i} \text { to } a_{j} \text { is not a path from } a_{e} \text { to } a_{j}\end{cases}
$$

Note that for $c_{i, j}=0$, there is either no wireless connection between $a_{i}$ and $a_{j}$, or $a_{i}$ to $a_{j}$ is not a path from $a_{e}$ to $a_{j}$. For a given routing algorithm, $C_{e}$ can be obtained if all link metrics are known. Let $m_{n}$ be the no. of mobile clients associating to $a_{n}$, and $\lambda$ be the incoming frame rate of the downlink traffic from the wired network to each client. We then define $t_{n}$ be the downlink traffic to $a_{n}$ 's clients. Therefore,

$$
t_{n}=\lambda m_{n}
$$

Let $h_{n}$ be the number of hops from $a_{e}$ to $a_{n}$, and $h_{\max }$ be the largest $h_{n}$ among all $n$. Define also $d_{i, n}$ be

$$
d_{i, n}= \begin{cases}1 & \text { if } h_{n}-h_{i}=1 \\ 0 & \text { otherwise }\end{cases}
$$

If $d_{i, n} c_{i, n}$ equals 1 , it implies that $a_{n}$ is a next hop of $a_{i}$ in the downlink transmission. 
Next, let $W_{i}$ be the bandwidth of the wireless link connecting $a_{i}$ and $a_{k}$ where $d_{k, i}=1$. Thus, the total carried traffic of each node, $f\left(a_{i}\right)$, can be obtained as:

$$
f\left(a_{i}\right)= \begin{cases}t_{i}+\sum_{n=1}^{N} f\left(a_{n}\right) c_{i, n} d_{i, n} & \text { if } i=e \\ t_{i}+\sum_{n=1}^{N} f\left(a_{n}\right) c_{i, n} d_{i, n} & \text { if } i \neq e \text { and }\left(t_{i}+\sum_{n=1}^{N} f\left(a_{n}\right) c_{i, n} d_{i, n}\right)<W_{i} \\ W_{i} & \text { if } i \neq e \text { and }\left(t_{i}+\sum_{n=1}^{N} f\left(a_{n}\right) c_{i, n} d_{i, n}\right)>W_{i}\end{cases}
$$

Note that eq. (4) is a recursive formula, as $f\left(a_{i}\right)$ depends on other $a_{n}$ where $i \neq n$. Therefore, we need to first find out $f\left(a_{n}\right)$ with $h_{n}=h_{\max }$. This is then followed by $f\left(a_{n}\right)$ with $h_{n}=h_{\max }-1, h_{\max }-2$, and so on. When $n=e$, we find the total carried traffic of the WMN at the MP $a_{e}$. The total carried traffic at the MP is limited when any one or more wireless links in the WMN are over-utilized, that is $f\left(a_{n}\right)>W_{i}$ for any $n$. By putting the MP in different places, we can obtain different values of the total carried traffic. The optimal placement of the MP is where $f\left(a_{e}\right) \geq f\left(a_{n}\right)$ for all $n \neq e$. Numerical examples will be given later to show how these equations can be used.

This study also gives the highest incoming frame rate that the network can support without over-utilizing any wireless links in the network. More specifically, we found it by increasing the value of $\lambda$ up to a value that the first wireless link in the network is over-utilized.

\subsection{Multiple MPs connecting to the wired network}

To increase the capacity and reliability of a network, multiple MPs are usually employed in a WMN. Let $E$ be the set of MPs in the WMN where $E \in A$. Given the routing algorithm, each MP in $E$ can calculate the shortest paths from itself to each MR in the network. We then compare the paths from each MP to the same MR. The shortest one among these paths is selected as the routing result and put into the matrix as Eq. (1). By considering all possible positions of MPs (i.e., $C^{N}$ numbers of combinations of MP positions are considered), we select the best positions of MPs that give the maximum total carried traffic. The total carried traffic is the sum of the carried traffic at each MP, i.e.,

$$
\text { Total carried traffic }=\sum f\left(a_{e}\right) \quad \text { where } e \in E
$$

\section{Numerical Examples}

The first example given below aims at finding the optimal placement of one single MP in a WMN. This WMN consists of six MRs namely $a_{1}$ to $a_{6}$ connecting one another. One of them will act as the MP of this network. As shown in Fig. 2, each node is assigned as the MP (the dark color node) alternatively, and the routing 
protocol RIP is then run to find the shortest paths from this MP to other MRs. The resulting shortest paths are shown in the dotted lines in the figure. According to these paths and Eq. (1), the routing results are represented in six matrices as given below.
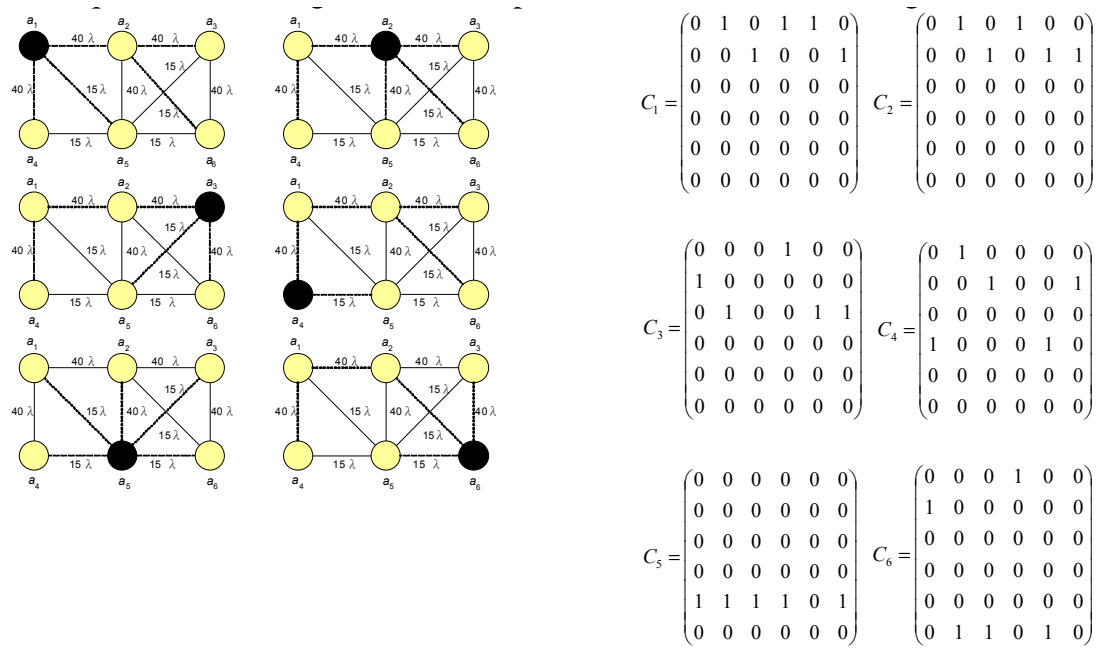

Fig. 2. RIP is running in a WMN with one MP at any position of six MRs

Assume that there are 20 numbers of mobile clients associating with each MR ( $m_{n}=20$ where $n=1,2, \ldots 6$ ). The incoming frame rates, $\lambda$, of the downlink traffic from the wired network to all clients are the same. It is further assumed that the frame processing rates of the MRs are always larger than the incoming traffic, that is, $\mu_{n} \geq \lambda m_{n}$ where $n=1,2,3 \ldots 6$.

According to Eq. (2), the traffic generated to each MR is,

$$
t_{n}=20 \lambda \quad \text { where } n=1,2, \ldots, 6 .
$$

Then, the total traffics aggregated at the MP in different locations are calculated by Eq. (4). Note that the capacities of the wireless links connecting among the MRs, $W_{i}$, are varied and limited to some values as shown on the links in the figure. Take $a_{1}$ be the MP as an example, its total aggregated traffic is calculated Eq. (7).

Eq. (7) is a recursive formula that $f_{R I P}\left(a_{3}\right)$ and $f_{R I P}\left(a_{6}\right)$ are needed to be found out first. It is because when $a_{1}$ is the MP, the largest number of hops (i.e., $h_{\max }$ ) will be 2 . And this happens when traffic is routed from the MP to either $a_{3}$ or $a_{6}$ (i.e., $h_{3}=h_{6}=2$ ). After obtaining $f_{R I P}\left(a_{3}\right)$ and $f_{R I P}\left(a_{6}\right)$, we continue to find $f_{R I P}\left(a_{2}\right), f_{R I P}\left(a_{4}\right)$, and $f_{R I P}\left(a_{5}\right)$ (because $h_{2}=h_{4}=h_{5}=h_{\max }-1=2-1=1$ ). Finally, $f_{R I P}\left(a_{1}\right)$ is obtained.

$$
f_{R I P}\left(a_{1}\right)= \begin{cases}t_{1}+\sum_{n=1}^{N} f\left(a_{n}\right) c_{1, n} d_{1, n} & \text { if } 1=e \\ t_{1}+\sum_{n=1}^{N} f\left(a_{n}\right) c_{1, n} d_{1, n} & \text { if } 1 \neq e \text { and }\left(t_{1}+\sum_{n=1}^{N} f\left(a_{n}\right) c_{1, n} d_{1, n}\right)<W_{1} \\ W_{1} & \text { if } 1 \neq e \text { and }\left(t_{1}+\sum_{n=1}^{N} f\left(a_{n}\right) c_{1, n} d_{1, n}\right)>W_{1}\end{cases}
$$


Table 1 shows the results for all possible placement of the MP. As seen from the second column of the table, if the MP of the WMN using RIP is placed at $a_{2}$, it can carry the highest volume of downlink traffic for the network $(115 \lambda)$.

Next, we consider applying another routing protocol HWMP to the above WMN. In our example, the HWMP is configured in the mode with root portal that most of the traffic is routed to the root (i.e., the MP). It is a kind of proactive routing protocol that uses a radio-aware routing metric to identify an efficient radio-aware path. The metric of each connection is represented by the number shown on the link as in Fig. 3. The paths having the minimum costs from the MP to other MRs are shown in the dotted lines while the costs are shown in the circles.
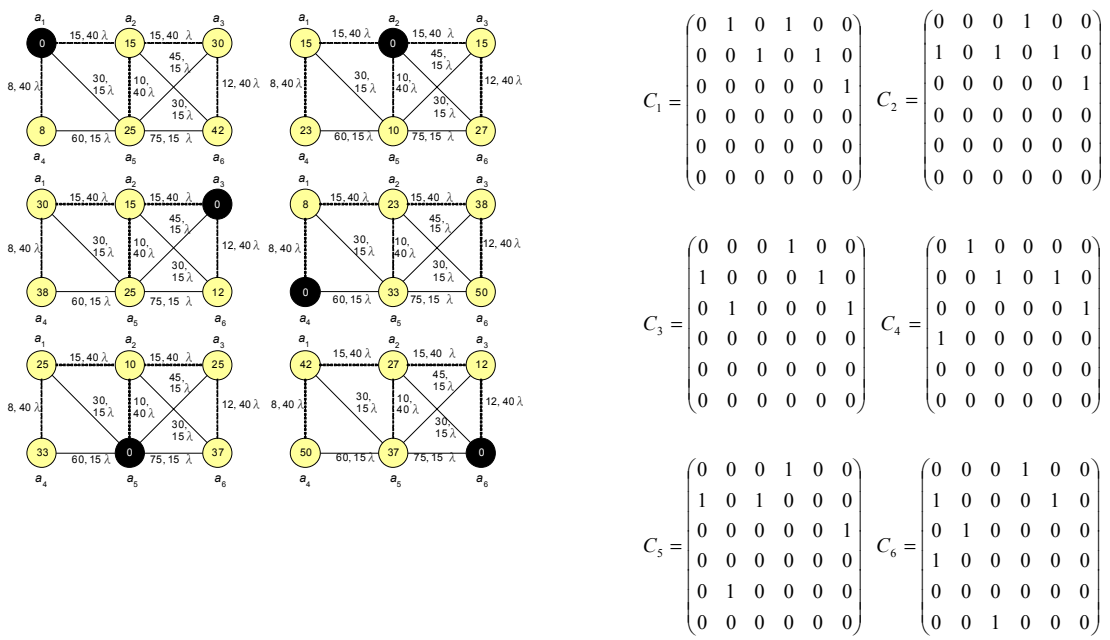

Fig. 3. HWMP is running in a WMN with one MP at any position of six MRs

By the same approach of calculating $f_{R I P}\left(a_{\mathrm{n}}\right)$, the total carried traffic at the MP located at each MR in the WMN running HWMP is calculated as shown in the last column of Table 1. As shown in the table, the MP of the WMN should also be placed at $a_{2}$ so as to support the largest downlink traffic (120 $\left.\lambda\right)$.

However, it is noted that the best performance of an algorithm may not be equal to that of another algorithm. From Table 1, the best performance of RIP can give $115 \lambda$ while that of HWMP can give $120 \lambda$. Therefore, a selection on routing protocol to the network is important.

On the other hand, if the MP is placed at $a_{4}$ of the network using RIP, it can only carry $75 \lambda$ downlink traffic. There is about $53 \%$ performance difference in placing the MP at $a_{2}$ and $a_{4}$. Similarly, there is $100 \%$ performance difference when HWMP is used. This indicates that the positions of MPs may influence the overall system throughput. 
Table 1. Total carried traffic at the single MP of the WMN

\begin{tabular}{llllll}
\hline MPs & $\boldsymbol{f}_{\boldsymbol{R I P}}\left(\boldsymbol{a}_{\boldsymbol{e}, f}\right)$ & $\boldsymbol{f}_{\boldsymbol{H W M P}}\left(\boldsymbol{a}_{\boldsymbol{e}, f}\right)$ & $\mathbf{M P s}$ & $\left.\boldsymbol{f}_{\boldsymbol{R I P}} \boldsymbol{a}_{\boldsymbol{e}, f}\right)$ & $\boldsymbol{f}_{\boldsymbol{H W M P}}\left(\boldsymbol{a}_{\boldsymbol{e}, f}\right)$ \\
\hline$a_{1}$ & $95 \lambda$ & $80 \lambda$ & $a_{4}$ & $75 \lambda$ & $60 \lambda$ \\
$a_{2}$ & $115 \lambda$ & $120 \lambda$ & $a_{5}$ & $100 \lambda$ & $60 \lambda$ \\
$a_{3}$ & $95 \lambda$ & $80 \lambda$ & $a_{6}$ & $70 \lambda$ & $60 \lambda$ \\
\hline
\end{tabular}

The second example considers that two MPs are deployed in the WMN. By running RIP again, the following matrixes for the routing results are obtained. Note that $C_{e, f}$ represents the case when $a_{\mathrm{e}}$ and $a_{\mathrm{f}}$ are selected as the MPs.

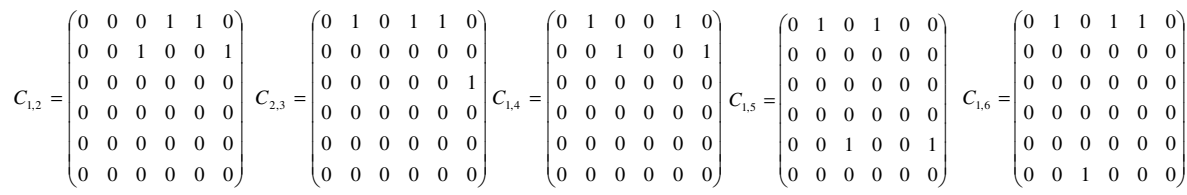

$C_{2,3}=\left(\begin{array}{llllll}0 & 0 & 0 & 1 & 0 & 0 \\ 1 & 0 & 0 & 0 & 1 & 1 \\ 0 & 0 & 0 & 0 & 0 & 0 \\ 0 & 0 & 0 & 0 & 0 & 0 \\ 0 & 0 & 0 & 0 & 0 & 0 \\ 0 & 0 & 0 & 0 & 0 & 0\end{array}\right) C_{2,4}=\left(\begin{array}{llllll}0 & 0 & 0 & 0 & 0 & 0 \\ 1 & 0 & 1 & 0 & 1 & 1 \\ 0 & 0 & 0 & 0 & 0 & 0 \\ 0 & 0 & 0 & 0 & 0 & 0 \\ 0 & 0 & 0 & 0 & 0 & 0 \\ 0 & 0 & 0 & 0 & 0 & 0\end{array}\right) C_{2,5}=\left(\begin{array}{llllll}0 & 0 & 0 & 0 & 0 & 0 \\ 1 & 0 & 1 & 0 & 0 & 1 \\ 0 & 0 & 0 & 0 & 0 & 0 \\ 0 & 0 & 0 & 0 & 0 & 0 \\ 0 & 0 & 0 & 1 & 0 & 0 \\ 0 & 0 & 0 & 0 & 0 & 0\end{array}\right) C_{2,6}=\left(\begin{array}{llllll}0 & 0 & 0 & 1 & 0 & 0 \\ 1 & 0 & 1 & 0 & 1 & 0 \\ 0 & 0 & 0 & 0 & 0 & 0 \\ 0 & 0 & 0 & 0 & 0 & 0 \\ 0 & 0 & 0 & 0 & 0 & 0 \\ 0 & 0 & 0 & 0 & 0 & 0\end{array}\right)$

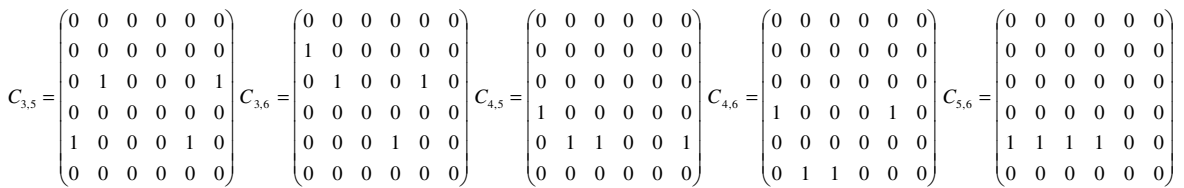

The total carried traffic at both MPs, $f^{\prime}{ }_{R I P}\left(a_{e, f}\right)$, is shown in the second column of Table 2. Then, we repeat the above steps for the WMN using HWMP and the results of $f_{H W M P}^{\prime}\left(a_{e, f}\right)$ are in the last column of Table 2.

Table 2. Total carried traffic at two MPs of the WMN

\begin{tabular}{|c|c|c|c|c|c|c|c|c|}
\hline MPs & $f^{\prime}{ }_{R I P}\left(a_{e, f}\right)$ & $f_{H W M P}^{\prime}\left(a_{e, f}\right)$ & MPs & $f^{\prime}{ }_{R I P}\left(a_{e, f}\right)$ & $f_{H W M P}^{\prime}\left(a_{e, f}\right)$ & MPs & $f^{\prime}{ }_{R I P}\left(a_{e, f}\right)$ & $f_{H W M P}^{\prime}\left(a_{e, f}\right)$ \\
\hline$a_{1}, a_{2}$ & $110 \lambda$ & $120 \lambda$ & $a_{2}, a_{3}$ & $115 \lambda$ & $120 \lambda$ & $a_{3}, a_{5}$ & $110 \lambda$ & $80 \lambda$ \\
\hline$a_{1}, a_{3}$ & $115 \lambda$ & $120 \lambda$ & $a_{2}, a_{4}$ & $115 \lambda$ & $120 \lambda$ & $a_{3}, a_{6}$ & $95 \lambda$ & $80 \lambda$ \\
\hline$a_{1}, a_{4}$ & $95 \lambda$ & $120 \lambda$ & $a_{2}, a_{5}$ & $110 \lambda$ & $120 \lambda$ & $a_{4}, a_{5}$ & $110 \lambda$ & $100 \lambda$ \\
\hline$a_{1}, a_{5}$ & $110 \lambda$ & $100 \lambda$ & $a_{2}, a_{6}$ & $120 \lambda$ & $120 \lambda$ & $a_{4}, a_{6}$ & $110 \lambda$ & $80 \lambda$ \\
\hline$a_{1}, a_{6}$ & $115 \lambda$ & $120 \lambda$ & $a_{3}, a_{4}$ & $115 \lambda$ & $120 \lambda$ & $a_{5}, a_{6}$ & $105 \lambda$ & $80 \lambda$ \\
\hline
\end{tabular}

Similar to the previous results, the placement of the MPs affects the system throughput. As shown in Table 2, the performance differences in placing the MPs at different positions are about $26 \%$ and $33 \%$ for the network RIP and HWMP respectively. Furthermore, the best positions of the MPs in the network using different 
routing algorithm may not be the same. For the example above, $a_{2}$ and $a_{6}$ are the best positions for the MPs of the network using RIP. This combination of MPs can support $120 \lambda$ downlink traffic. However, for the network using HWMP, $a_{1}$ and $a_{2}, a_{1}$ and $a_{3}$, $a_{1}$ and $a_{4}, a_{1}$ and $a_{6}, a_{2}$ and $a_{3}, a_{2}$ and $a_{5}, a_{2}$ and $a_{6}$ or $a_{3}$ and $a_{4}$ can also be the best positions of the MPs.

\section{Conclusion}

In this paper, we show that the placement of $\mathrm{MP}(\mathrm{s})$ in a WMN is important that may affect the network throughput of the network. Numerical examples show that there is up to $100 \%$ throughput difference in placing a MP at different positions. Also, the best positions of MPs in a network using different routing algorithms may not be the same. Therefore, to compare the routing algorithms by a given set of MP positions is not fair. Instead, the optimal performance of the routing algorithms can be found by finding the corresponding optimal placement of MPs first. Lastly, a selection in routing algorithm is still a key performance factor of the network because the best performance for an algorithm may not be equal to that of another algorithm.

\section{References}

1. Akyildiz, I.F., Wang, X.: A Survey on Wireless Mesh Networks. IEEE Radio Communications 43(9), S23 - S30 (2005)

2. Wu, X., Liu, J., Chen, G.: Analysis of Bottleneck Delay and Throughput in Wireless Mesh Networks. IEEE MASS, 765-770 (2006)

3. Jun, J., Sichitiu, M.L.: The Nominal Capacity of Wireless Mesh Networks. IEEE Wireless Communications 10, 8-14 (2003)

4. Gupta, P., Kumar, P.R.: The Capacity of Wireless Networks. IEEE Transaction of Information Theory 6(2), 388-404 (2000)

5. Gamal, A.E., Mammen, J., Prabhakar, B., Shah, D.: Throughput-delay Trade-off in Wireless Networks. In: Proc. IEEE INFOCOM (2004)

6. Zhang, H., Tsang, D.H.K.: Traffic Oriented Topology Formation and Load-balancing Routing in Wireless Mesh Networks. In: Proc. IEEE ICCC, pp. 1046-1052 (2007)

7. Chandra, R., Qiu, L., Jain, K., Mahdian, M.: Optimizing the Placement of Internet TAPs in Wireless Neighborhood Networks. In: ICNP, pp. 271-282 (2004)

8. Li, F., Wang, Y., Li, X.Y.: Gateway Placement for Throughput Optimization in Wireless Mesh Networks. In: Proc. IEEE ICC, pp. 4955-4960 (2007)

9. Draves, R., Padhye, J., Zill, B.: Comparison of Routing Metrics for Static Multi-hop Wireless Networks. In: Proc. of the ACM SIGCOMM (2004)

10. Shen, Q., Fang, X.M.: A Multi-metric AODV Routing in IEEE 802.11s. In: Proc. IEEE ICCT, pp. 1-4 (2006)

11. Zhu, C.H., Lee, M.J., Saadawi, T.: On the Route Discovery Latency of Wireless Mesh Networks. In: Proc. IEEE CCNC, pp. 19-23 (2005)

12. Liu, L.G., Feng, G.Z.: Mean Field Network Based QoS Routing Scheme in Wireless Mesh Networks. In: Proc. IEEE WCMN, vol. 2, pp. 1110-1113 (2005) 